\title{
What contributions of Artificial Intelligence in Innovation?
}

\author{
Yassine Barakat ${ }^{1, *}$, Salmane Bourekkadi ${ }^{2}$, Samira Khoulji ${ }^{1}$, and Mohamed Larbi Kerkeb ${ }^{1}$ \\ ${ }^{1}$ The Information Systems Engineering Research Team (ERISI), National School of Applied Sciences - Abdelmalek Essaadi University, \\ Tetouan-Morocco, \\ ${ }^{2}$ Ibn Tofail University, Kenitra, Morocco
}

\begin{abstract}
Artificial intelligence has proven its effectiveness through many applications in society: medical diagnostics, e-commerce, robot control and remote sensing. It has been able to advance many fields and industries including finance, education, transportation and others. In this review article, the method adopted consists first of all in collecting and analyzing all the data making it possible to certify the existence of a varying impact of artificial intelligence on the environment and the sustainable development, through the description of the contributions of AI at the level of several approaches carried out and projects carried out recently. Secondly, we drew from the different levels of differential paradigms cited in the first part, a synthesis, a critical point of view, as well as the perspectives that the feedbacks to the various points raised in the critical vision create, in particular those related to the limits of artificial intelligence and its implications for sustainable development.
\end{abstract}

\section{Introduction}

Nowadays, Artificial Intelligence (AI) flourishes in a daily basis in both, the public sector (administrations, research centers, universities, etc...) and the private sector (companies, design offices, etc...). Artificial Intelligence is defined as machines or programs capable of performing tasks that are normally carried out by human intelligence (Marvin Minsky,2019): This includes calculating, learning and adapting to new situations, processing very large data sets, making predictions, all based on machine learning and deep learning.

Using relatively basic equipment to train deep learning models in a reasonable amount of time. These models are based on multilayer neural networks and can learn to represent high level information of data abstraction. A classic (supervised) deep learning process (see figure 1) is as follows:

- Select the network architecture according to the task to be performed ;

- Train several models to test them and optimize the parameters ;

- Test each model on other data, which will evaluate their actual performance.

Many neophytes instinctively oppose progress and nature, science and environment. So what impact of artificial intelligence on the environment?

At first look, it may not seem obvious to the general public, but artificial intelligence technology can make a positive contribution to the environment. As underlined in particular by our article, the main goal of which is, to compile a set of data, studies and experiences affirming this positive correlation between $\mathrm{AI}$ and sustainable development.

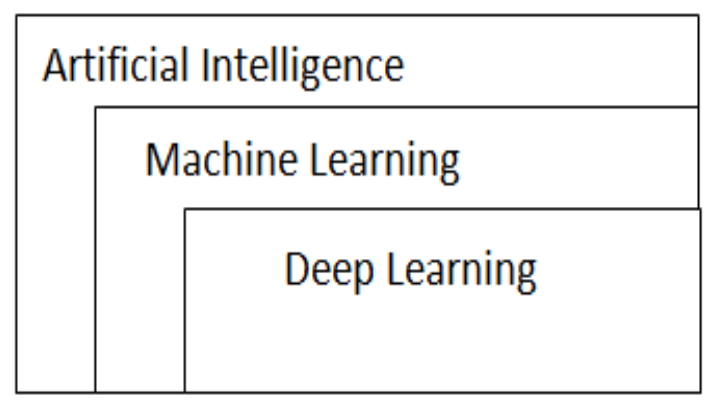

Fig.1. Artificial Intelligence, Machine and Deep Learning.

\section{Data Collection And Analysis}

Sustainable development can benefit from artificial intelligence (Peter Addo,2020), in particular by optimizing energy and transport networks, improving the efficiency of certain activities (such as agriculture, building management and urban development) and by making more precise forecasts to increase the potential (such as Climate change, agricultural productivity, biodiversity) and support for decision-making.

\subsection{Al And Renewable Energies}

There are many applications of artificial intelligence, which can save tens of billions of dollars in expenses by using Machine learning technics, algorithms and predictive models. The potential of artificial intelligence applications in the development of renewable energies is

\footnotetext{
* Corresponding author: yassine.barakat@gmail.com
} 
so great that many players in this market are testing innovative solutions to increase the production of different systems. Artificial intelligence applications can help to fully exploit the equipment by predicting the weather and the operating conditions, such as identifying best sun exposition for the photovoltaic surface exposed, the wind direction and power, and rainfall index of hydroelectric power generation. However, they can also help manage the energy supply of families living in cities by optimizing the entire distribution network.

\subsection{Al and Solar / Wind Energy}

The use of artificial intelligence improves energy management by optimizing traffic (Géraldine Marcheteau ,2020). For example, this makes it easier to predict peaks in consumption while taking into account the share of energy produced. Indeed, the technological development has allowed the use of artificial intelligence smart grid in the field of energy. The smart grid is equipped with various technological artificial intelligence solutions. It is about creating an intelligent network, where there is a balance between supply and demand, even if there is a peak in consumption. Thanks to the installation of smart meters in homes, for example, it is possible to monitor electricity consumption in real time. These data allow professionals to forecast energy production and distribution at peak times, while integrating green energy generated by individuals: it is essential to improve the energy efficiency of the entire network. AI has an important role in the creation of autonomous energy micro networks, this principle is explained by our following:

An "electrically" autonomous village draws its energy from a wind turbine and solar panels. Autonomy that also involves a bank of batteries, charged batteries for days without sun or wind. And finally, a diesel generator when nothing is working to optimize everything, we add a touch of artificial intelligence( see figure 2).

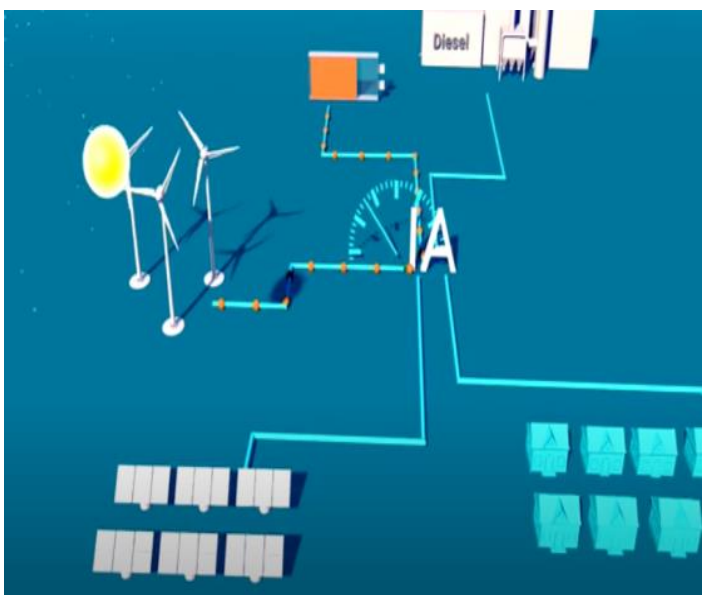

Fig. 2. Battery charging case.

Although they are inexhaustible, renewable energies have a big flaw: they fluctuate according to the force of the winds and the periods of sunshine, this energy, is not stable. And since it is not stable, so it can disrupt the network.
Artificial intelligence can contribute in the argumentation of what is called the stability of the network or in the robustness of the network because it can analyze in continuity, in real time and in a few fractions of a second the quality of electricity provided.

For example, we have a village where energy sources are interconnected and converge towards artificial intelligence. Everything is designed to prioritize the use of renewable energies and to minimize the use of fossil fuels, such as diesel generators.

- we can observe the interest of artificial intelligence in a hybrid micro network, multi source, at the level of energy flow management.

So how can we distribute energy and power between different sources and different loads?

- it is 12 o'clock (see figure 3). the sun is hot, the wind is blowing. Artificial intelligence stores the energy produced by the wind turbine, it considers that the solar panels are sufficient to supply the village with energy.

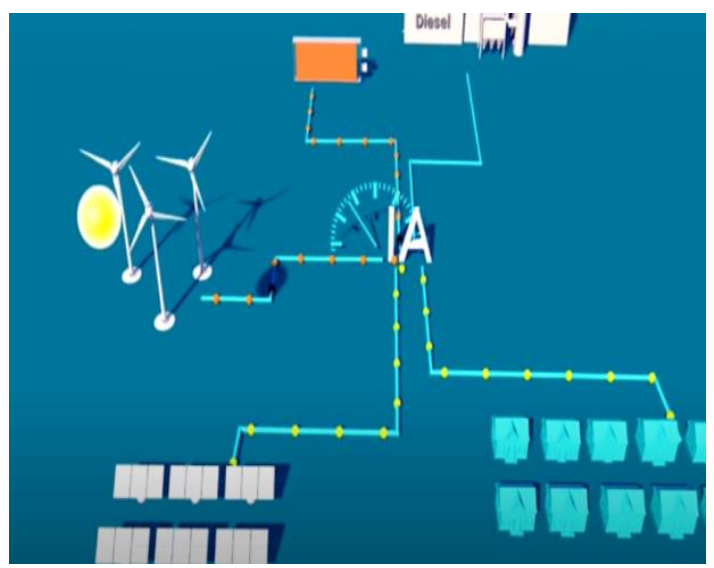

Fig. 3. 12hr case.

- It is 6 p.m (see figure 4). the demand for energy is exploding, but the clouds have appeared. No problem. Artificial intelligence taps into the battery park.

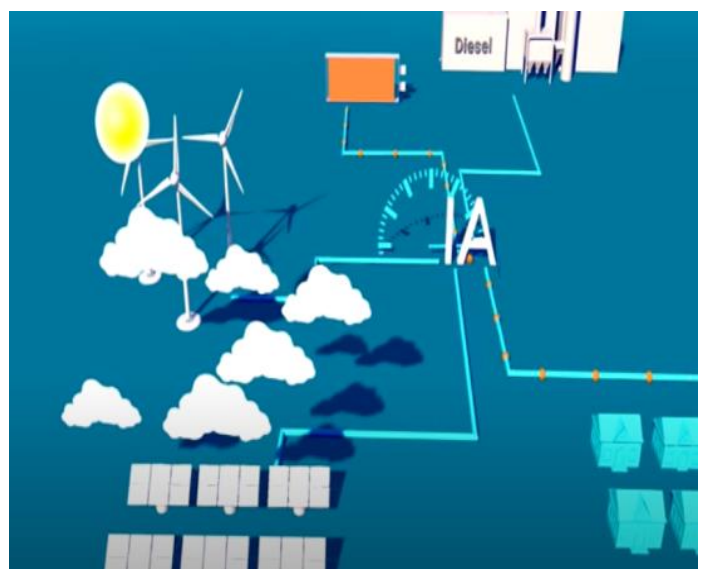

Fig. 4. $18 \mathrm{hr}$ case.

To develop such consumption strategies, artificial intelligence must have an imposing computing power.

For example in a wind turbine, we can fetch 200 information per second (Hussein Ibrahim,2018). So, if we want to process these 200 pieces of information per second, we can't, our brain will burst. So we need intelligent algorithms that process this information. 
- To optimize the network, the algorithms at the base of artificial intelligence must be fed with a large amount of various data. «These are operational data such as parameters, such as voltage, frequency, oil pressure, air pressure, temperature, flow rates, flows, etc. » adds Hussein Ibrahim.

Artificial intelligence must also anticipate the habits of residents: preparing meals, washing, all depending on the vagaries of the weather and the performance of green energy sources.

\subsection{Al And Sustainable Development: The Case Of Electronics And Agriculture}

Artificial intelligence can facilitate the choice of projects that eliminate waste in the food sector (Pascal Decaux, 2019) : artificial intelligence technology can indicate when fruits and vegetables can be harvested and adjust accordingly. During a given period, food products are continuously supplied according to demand. Beneficial Mechanism Can Save Almost US \$ 130 Billion Per Year by 2030 (Ellen MacArthur Foundation, Artificial intelligence and the circular economy - AI as a tool to accelerate the transition ,2019). in the field of consumer electronics, artificial intelligence can use predictive models and improve equipment maintenance. Intelligently manage e-waste recycling system using image recognition and robot mechanisms. By 2030, these apps can save up to $\$ 90$ billion, the report continues.

This is the case of the city of Dubai, which has implemented a plan to use artificial intelligence to optimize the collection and recycling of waste (scient.fr,2019). Once the plan is in place, a decision support algorithm is used to screen the entire waste treatment process, which will provide recommendations for reducing operating and environmental costs. The project also includes the construction of a waste recycling plant to produce $100 \%$ environmentally friendly concrete, on the other hand, converting the energy released during the recycling process into green electricity to supply energy to the city.

\subsection{Al and Agriculture}

In the agricultural sector, most of the focus at the moment is machine learning. In order to provide "smart" algorithms, the first step is to collect large amount of data. Thanks to sensors installed on land or on tractors, cameras and even soil mapping using drones. Then this data is sent to the cloud, where it can be crushed by algorithms. We use the cloud because there is too much data and a lot of computing power is needed to process it, this power can be obtained by mobilizing many servers in the cloud.

Analyzing this data will allow farmers to make informed decisions based on large amount of data (analyzed by machine learning algorithms). Thus, the "smart farmer" has all the information necessary to make key choices: the date of sowing, harvesting or adding phytosanitary products (fertilizers, pesticides), the rate of irrigation, etc. We can monitor everything and then analyze it. Through AI, to promote agricultural work.
By analyzing the image of the soil and the farms, applications and tools such as Carbon Bee, Exotic Systems, VisioGreen, Weenat, the Karnott box developed by the startup ConnectAgri, PEAT, Plantix from Trace Genomics or the sensor tag from Yes it is , provide farmers with precise analysis and recommendations to optimize their productivity.

Thus the use of drones with machine learning and deep learning make it possible to analyze the data in an ever more precise way in order to give results such as the height of the plants, the condition of the seeds, analyzes of the fertilization or even measurements of hydrometry (usbek\&trica, 2018). For example, the Plantix deep learning application, developed by the Berlin start-up PEAT, works as an image recognition application: after analyzing the foliage of plants, its algorithms are able to establish a correlation with certain soil defects, the presence of pests or a disease (see figure 5).

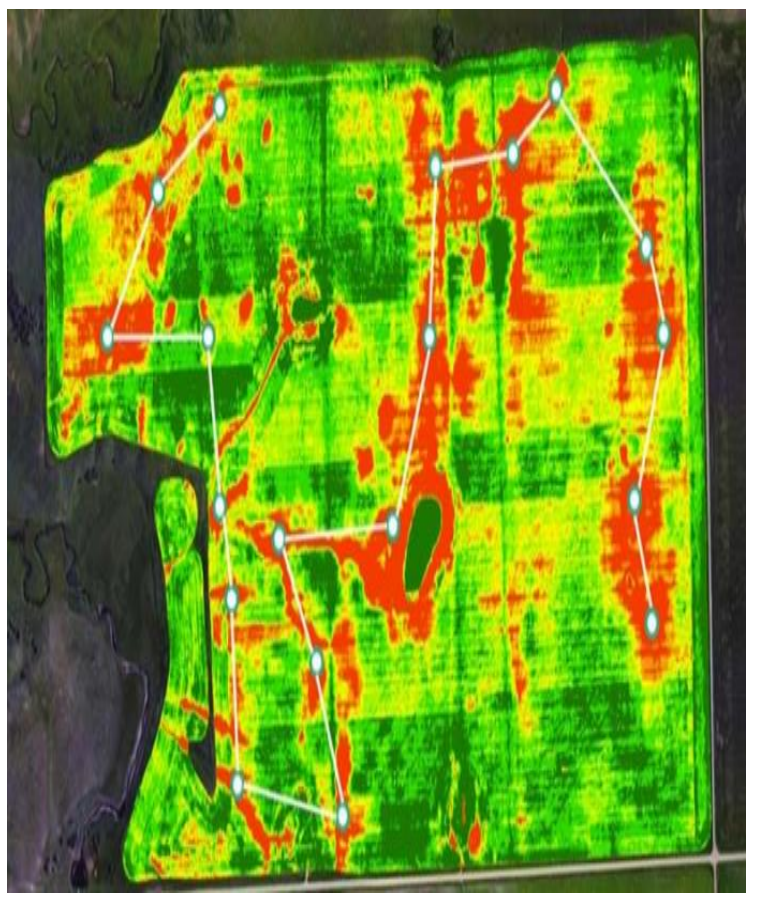

Fig. 5. Weed path by PLANTIX.

We add that with AI and robotics, we are able to identify when the product is ripe as well to catch them and we can use less pesticides, less weed killers and especially less fertilizer by the use of small robots that will walk on the fields, for example to spot the weed and to attack it.

\subsection{Al and ocean}

Others use artificial intelligence to protect the ocean. In many current projects, we can cite the "Soft Robotic Fish" (see figure 1) set up by researchers from the Massachusetts Institute of Technology. It is a robotic fish, and scientists use it to observe marine life in its natural ecosystem without scaring them away. Therefore, it is possible to study the effects of marine pollution on the behavior of fish. Currently, robotic fish can be controlled remotely, but the ultimate ambition is to let it use artificial intelligence to automatically track a bench of fish. It's up to creating a self-sustaining fish like a real 
fish that can automatically adapt to the life of the sea life without human involvement.

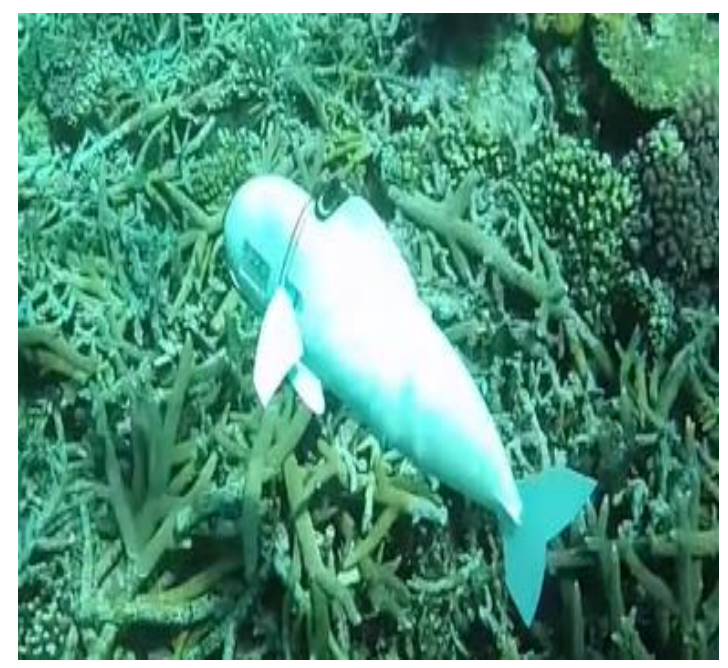

Fig. 6. Soft Robotic Fish in the sea

Other initiatives use robotics to solve plastic pollution in the ocean. This is the case of Project "the ocean cleanup", which mobilizes robots 600 meters long capable of collecting up to 5 tons of plastic per month (Microsoft experiences, 2019). The robot, called Wilson, can be controlled remotely via a satellite that communicates with the Ocean Cleanup headquarters and cameras. The first robot was deployed in early September 2019. Ultimately, the objective is to constitute an equipment team made up of 60 pieces of equipment and to recycle the collected plastic. Another program called "The Plastic Tide Project" uses drones, the mission of which is to fly over certain areas to find plastic waste (Joe McCarthy, 2018). They use machine learning algorithms to identify litter in the ocean.

\section{Interpretation of results, discussion and critical vision}

Thus, artificial intelligence plays an important role in optimizing energy consumption by using smart meters allowing energy producers and distributors to read and analyze energy consumption at each installation location. (c.-à-d. Apartments, houses, shops, town halls, schools, swimming pools, etc.). For its part, customers (individuals, businesses, communities, etc.) can monitor their consumption by connecting to mobile applications or websites, thus controlling their consumption. It is therefore possible to remotely manage the temperature of the room or even turn off the lights if the premises are empty.

In the United States, utility companies can remotely reduce customer energy consumption by connecting to dedicated services (Charles Beneteau, 2020), thereby reducing peak loads. By controlling overall energy consumption, the infrastructure investment required to increase maximum load capacity is reduced.

Thanks to technological progress, more and more farmers can practice precision agriculture, which not only takes into account the data extracted from each agricultural region and each land, but also takes into account the heterogeneity of cultivated land. Thus, they can adapt their actions to the different ecosystems they encounter.

Analysis of soil and farm health is the main problem for farmers. Machine learning and deep learning provide new tools for farm monitoring, especially in the analysis of soil samples, which can more easily detect possible contamination, microbial reproduction and even harmful genetic mutations. For example, algorithms, image recognition and computer vision make it possible to use a simple smartphone to recognize real-time status and needs.

The development of artificial intelligence is rapidly increasing the demand for electronic equipment (cpu, robot, etc.). To manufacture this material, industry needs rare metals (or rare earths). However, the extraction and refining of rare metals results in the release of many toxic elements into the environment: heavy metals, sulfuric acid, radioactive elements, etc.

In addition, at the end of their life cycle, this electronic equipment generates large amounts of waste (electronic waste; 50 million tonnes per year worldwide (Davos, Suisse (OIT Infos), 2019)). Only 20\% of this waste is properly recycled. The rest are incinerated, thrown away or sent to countries that do not have the capacity to recycle this waste safely. In the latter case, the equipment was disassembled by hand, exposing these people to toxic products.

\section{Al's energy and ecological footprint}

It is estimated that by 2025 , the digital ecosystem will consume up to $20 \%$ of the world's electricity and will generate $5-8 \%$ of carbon emissions (Hugo Pellegrin, 2019) (more than aviation and transport). A recent study estimated that training a single AI (deep learning) system would emit up to 284 tons of carbon (Donna Lu, 2019), i.e. five times more than the entire life cycle of a car (including its life cycle), including its manufacture. Designers are working to improve the energy efficiency of the digital ecosystem, but as products and services continue to increase, these benefits are offset: more data to store and circulate, more devices, more algorithms, etc.

Artificial intelligence requires machines to function, these machines are made up of equipment and materials (rare metals for batteries) that have caused controversy. However, the most frequently mentioned is the power consumption of these devices and the energy lost to cool everything (lethal energy). When calculations are needed, data is written, read or copied to the data center, the computer "heats up". And although we are trying to find components that consume less power, they still need to be cooled in order to function properly.

Air conditioning generally cools everything, which will also consume electricity, and paradoxically heat "elsewhere", outside of data centers. We end up with an increased "external" heat. Hence the need to work on cooling solutions by immersing, for example, servers in liquids or underwater bases ... but the problem of heat remains the same: it disperses outside. Thus AI is indirectly a main cause of the emission of a significant amount of co2, according to some researchers (Emma 
Strubell, Ananya Ganesh, Andrew McCallum, 2019) who have studied the life cycles of several models of Most popular AI («Deformable model », ELMo, BERT, ORET, GPT-2, etc.). They train them on a single microprocessor each day from large amounts of data to calculate the amount of electricity needed, then multiply the result by the number of hours and the microprocessor used in the machine. Artificial Intelligence research framework. Thus, they were able to estimate the carbon dioxide equivalent consumed by the deep learning method based on the average energy structure of the United States (17\% renewable energy, 35\% natural gas, $27 \%$ coal and $19 \%$ nuclear energy).

According to their research, although a person's plane from New York to San Francisco consumes the equivalent of a ton of CO2 (1984 pounds), some trialand-error algorithm learning models have been generated. In a few days, the $\mathrm{CO} 2$ equivalent is between 78,468 to 626,155 pounds. For reference, the average person produces 11,023 pounds of $\mathrm{CO} 2$ equivalent per year. A car will produce 126,000 pounds of $\mathrm{CO} 2$ equivalent in the same amount of time. In other words, the life of a deep learning model trained for 4-7 days is equivalent to a 57 years of humans life, or 5 cars produced in a lifetime.

\section{Conclusion}

Artificial intelligence has great potential for sustainable development, in particular by optimizing energy and transport networks, improving the efficiency of certain activities in agriculture, building management and urban development. But we must not forget that AI has certain flaws that are harmful to the environment and for which we must seek sustainable solutions.

Artificial intelligence needs to be fed quality training data before it is operational, but this data is not always accessible or affordable at low cost. However, the insufficient quality of training data, in terms of diversity or representation, can lead to distortions, discrimination or erroneous conclusions which will not contribute to the achievement of development objectives. In addition, failure to incorporate ethical requirements into machine learning algorithms poses risks to development projects.

Finally, the issues related to data confidentiality should not be underestimated. The general data protection regulation could slow down the development of AI development if data protection principles are not well respected. Yet developing AI systems that incorporate privacy from its conception is often expensive. The upstream involvement of data protection authorities can help alleviate this problem. In this regard, methods that allow AI models to protect data privacy are preferred.

Within five years, given the rapid pace of developments in artificial intelligence, models currently considered to be a review will have become obsolete. The next generation of artificial intelligence, employing novel approaches to AI such as unsupervised learning, federated learning, and the Transformer model, will open up currently unimaginable possibilities in technology, and therefore development.

\section{References}

1. P. Emond,Etienne. L'intelligence artificielle et l'environnement: un mariage naturel.LEDEVOIR,(2019), number 7 DE 10,2-3

2. D. Lu,Créer une IA peut être cinq fois pire pour la planète qu'une voiture,newscientist,2019, number 3234

3. E. Strubell and A. Ganesh and A. McCallum, Energy and Policy Considerations for Deep Learning in NLP,(2019),abs/1906.02243

4. Evaluating the Energy Efficiency of Deep Convolutional Neural Networks on CPUs and GPUs, Li et al., IEEE International Conferences on Big Data and Cloud Computing (BDCloud), Social Computing and Networking (SocialCom), Sustainable Computing and Communications (SustainCom)(BDCloud-SocialCom-SustainCom),( 2016)

5. Energy and Policy Considerations for Deep Learning in NLP, Strubell et al., ACL (2019)

6. Green AI, Roy Schwartz et al., (2019), https://arxiv.org/abs/1907.10597

7. Comprendre l'intelligence artificielle, Nicolas Sabouret, dessins de Lizete De Assis, Ellipses, (2019)/

8. Ellen macarthur foundation. Artificial intelligence and the circular economy. mckinsey. (2019)

9. Baldé, C. P., Forti, V., Gray, V., Kuehr, R., Stegmann, P., The Global E-waste Monitor (2017): Quantities, Flows, and Resources (2017)

10. Marr, B., How Much Data Do We Create Every Day? The Mind-Blowing Stats Everyone Should Read, Forbes (2018)

11. ActuIA.L'intelligence artificielle dans le domaine maritime. available on $<$ https://www .actuia.com/actualite/journeemondiale-de-locean-quelques-exemples-dutilisationde-lia-dans-le-domaine-maritime/>. Octobre2020)

12. Usbek\&Rica.Quand l'intelligence artificielle vole au secours du climat. available on: $<$ https://usbeketrica.com/fr/article/l-ia-peut-ellesauver-la-planete>. (12 Octobre 2020).

13. Jean-David Benassouli.Bâtir un avenir durable grâce à l'intelligence artificielle. available on: $<$ https://www.pwc.fr/fr/publications/data/batiravenir-durable-grace-a-intelligenceartificielle.html>. (15 octobre 2020)

14. Microsoft. L'IA peut-elle sauver la planète. available on: $<$ https://experiences.microsoft.fr/business/intelligen ce-artificielle-ia-business/ia-peut-elle-sauver-laplanete/>. (20 Octobre 2020)

15. C. Ingrand. Environnement : l'intelligence artificielle laisse-t-elle une empreinte carbone bien réelle. available on : <https://www.lci.fr/hightech/intelligence-artificielle-ia-une-empreintecarbone-co2-bien-reelle-algorithme-machine- 
learning-environnement-cloud-2123439.html >.(20 septembre 2020)

16. Gouvernement du Quebec : Commission de l'éthique en science et en technologie.Intelligence artificielle et environnement : alliés ou ennemis. available on: <Gouvernement du Quebec : Commission de l'éthique en science et en technologie $>$. (30 aout 2020)

17. GNITEKRAM.Sauver la planète grâce à l'intélligence artificielle. available on: $<$ http://gnitekram.fr/sauver-la-planete-grace-alintelligence-artificielle/>. (25 octobre 2020)

18. Cédric Vasseur. L'intelligence artificielle, un bienfait pour la planète ou un risque écologique. available on: <https://blog.orsys.fr/lescarnets/index.php/2020/01/23/lintelligence-

artificielle-un-bienfait-pour-la-planete-ou-un-risqueecologique/>(26 octobre 2020)

19. Géraldine Marcheteau,l'intelligence artificielle dans la gestion de l'énergie,2018, available on: $<$ https://www.lenergietoutcompris.fr/actualitesconseils/comment-utiliser-1-intelligence-artificielledans-la-gestion-de-1-energie-48330>. (29 octobre 2020)/.

20. Julien MERCIER.L'intelligence artificielle au service du développement durable.construction 21. (2018)

21. Microsoft. IA \& Machine Learning : quels bénéfices pour l'agriculture. available on: $<\mathrm{https}$ ://experiences.microsoft.fr/business/intelligen ce-artificielle-ia-business/intelligence-artificielleagriculture >. (01 novembre 2020)

22. ActuIA.Quel sera l'impact de l'intelligence artificielle sur l'agriculture. available on: $<$ https://www.actuia.com/dossiers/sera-limpact-delintelligence-artificielle-lagriculture/>. novembre2020)

23. Usbek\&Rica.Comment l'intelligence artificielle transforme l'agriculture. available on: $<$ https://usbeketrica.com/fr/article/comment-lintelligence-artificielle-transforme-l-agriculture-1>. (11 Novembre 2020). 